Jahrbuch Schweiz - Dritte Welt 1996

\title{
Eine soziale Weltwirtschaft braucht internationale Sozialklauseln
}

Zehn Thesen aus Sicht der Wirtschaftsethik

\section{Christoph Stückelberger}

\section{(2) OpenEdition}

\section{Journals}

Electronic version

URL: http://journals.openedition.org/sjep/1345

DOI: $10.4000 /$ sjep.1345

ISSN: 1663-9677

\section{Publisher}

Institut de hautes études internationales et du développement

Printed version

Date of publication: 1 mars 1996

Number of pages: $241-248$

ISSN: 1660-5926

\section{Electronic reference}

Christoph Stückelberger, «Eine soziale Weltwirtschaft braucht internationale Sozialklauseln », Schweizerisches Jahrbuch für Entwicklungspolitik [Online], 15 | 1996, Online erschienen am: 17 Mai 2013, abgerufen am 08 September 2020. URL : http://journals.openedition.org/sjep/1345; DOI : https:// doi.org/10.4000/sjep.1345 


\title{
Eine soziale Weltwirtschaft braucht internationale Sozialklauseln
}

\section{Zehn Thesen aus Sicht der Wirtschaftsethik}

\author{
Christoph Stückelberger
}

„Die Gemeinschaft wird immer mehr fragen: Was dient dem Ganzen? Die zügellose Freiheit des einzelnen, wodurch im Grunde ganze Massen unfrei werden, wird immer mehr weichen müssen der Freiheit des Ganzen. Der Staat wird unter anderem ein Fabrikpolizeigesetz aufstellen. ... Endlich wird der Staat auf ein internationales Gesetz hinzuwirken suchen. Erst wenn ein Gesetz aller oder doch der grössten industriellen Staaten, das dann die verbindliche Norm für jede Partikulargesetzgebung wäre, die Schulbildung der Kinder verlangt, ihre allzufrühe Anstellung und die rücksichtslose Ausbeutung ihrer Arbeitskraft auf Kosten ihrer leiblichen und geistigen Ausbildung verhindert, das übermässige Arbeiten verbietet, für den gebrochenen, kranken und betagten Arbeiter sorgt, erst dann ... hören diese Schäden der Fabrikindustrie auf."

Dieses Zitat stammt nicht aus einer Rede bei den GATT-Verhandlungen der letzten Jahre, sondern aus der Schrift "Ein Wort über die Fabrikindustrie“ 
von $1858 !^{1}$ Der Autor Bernhard Becker war Pfarrer im kleinen Bergkanton Glarus in der Schweiz, wo 55 Prozent der arbeitenden Bevölkerung in der Baumwollindustrie tätig waren und viele wegen Hunger als „Wirtschaftsflüchtlinge" nach Amerika auswanderten. Becker war ein Urheber des Fabrikgesetzes des Kantons Glarus, des ersten Fabrikgesetzes in der Schweiz und des ersten Fabrikgesetzes Europas, das den Arbeitstag auf 12 Stunden beschränkte. Pfarrer Becker vertrat schon vor 140 Jahren die Auffassung, dass es nationale sowie auch internationale gesetzliche Vereinbarungen braucht, um eine soziale, menschengerechte Ordnung der Wirtschaft zu erlangen!

Damit setzte die lange Bewegung hin zur sozialen Marktwirtschaft ein, die von der protestantischen Ethik deutlich mitgeprägt war ${ }^{2}$. Die Debatte um die Erreichung resp. Sicherstellung der sozial gerechten Verteilung der Errungenschaften der Marktwirtschaft sind heute national wie international durch die Liberalisierung des Welthandels wieder aktuell. Die weltweite Einhaltung sozialer Mindeststandards im Arbeitsbereich durch Sozialklauseln ist ein wesentlicher Teil der heutigen Suche nach einer menschenwürdigen Entwicklung. Mit Sozialklauseln werden im folgenden Bestimmungen für soziale (bes. arbeitsrechtliche) Minimalstandards verstanden, die im internationalen Handel zu respektieren sind und mit denen positive oder negative Massnahmen gegenüber jenen ergriffen werden können, die diese Minimalstandards nicht einhalten.

Zehn ethische Gründe für solche Sozialklauseln seien in Kurzform genannt.

\section{Menschenwürdige Entwicklung für alle ist das Ziel der Wirtschaft}

Was ist eigentlich das Ziel wirtschaftlicher Tätigkeit? Diese Grundfrage muss immer wieder hartnäckig gestellt werden. Kapitalvermehrung, ökonomisches Wachstum oder Sieg über die Konkurrenz sind nicht Selbstzweck, höchstens Mittel zum Ziel. Zu welchem Ziel? Alle Menschen, ja alle Lebewesen haben das Grundbedürfnis zu leben, zu wachsen und sich zu entfalten. Sie haben theologisch gesprochen - von Gott das Geschenk eines Lebens in Würde zugesprochen erhalten. Ziel wirtschaftlicher Tätigkeit ist es, durch Produktion und Verteilung von Gütern und Dienstleistungen ein Leben in Würde für möglichst viele Menschen und nichtmenschliche Kreaturern zu ermöglichen.

1. Becker, B.: Ein Wort über die Fabrikindustrie (1858). Mit einer Einführung von H.-U. Schiedt, hg. vom Institut für Sozialethik des SEK, Bern 1990. Hervorhebung durch den Verf.

2. Vgl. Brakelmann, G./ Jähnichen, T.: Die protestantischen Wurzeln der sozialen Marktwirtschaft. Ein Quellenband, Gütersloh 1994: „Wir stellen die zentrale These sofort voran: Das Konzept 'soziale Marktwirtschaft' ist in wesentlichen Zügen von den sozialethischen Traditionen des Protestantismus mitbestimmt." (Einleitung, 13) 
Das Wirtschaften soll eine menschenwürdige Entwicklung weltweit ermöglichen. Dass unter einer menschlichen Entwicklung nicht nur die Erhöhung des Bruttosozialproduktes zu verstehen ist, zeigen z.B. der „Human Development Index" HDI des UNO-Entwicklungsprogramms UNDP oder Indices wie jene von Daly/Cobb ${ }^{3}$.

\section{Die Menschenrechte sind die Grundlage sozialer Entwicklung}

Die „Allgemeine Erklärung der Menschenrechte“ wie die UNO-Pakte über politische, wirtschaftliche, soziale, kulturelle Rechte und das „Recht auf Entwicklung“ bilden (trotz aller Diskussionen über die universelle Gültigkeit dieser Rechte) den weltweit weitgehend anerkannten Massstab zur Beurteilung menschenwürdiger Entwicklung. Die wirtschaftliche Entwicklung und der Welthandel als wesentlicher Teil davon sind an diesem Massstab zu messen. Bei jeder wirtschaftlichen Massnahme ist zu fragen: Fördert oder erschwert sie eine menschenwürdige, soziale Entwicklung und die Respektierung der Menschenrechte? Sozialklauseln haben dabei den Rechten der Frauen besondere Beachtung zu schenken, denn Frauenrechte sind Menschenrechte. Sozialklauseln zielen darauf ab, die Verletzung von Menschenrechten weltweit zu bekämpfen. Die für eine Sozialklausel vorgesehenen sieben ILOKonventionen basieren auf solchen grundlegenden Menschenrechten. Sechs der sieben (Ausnahme: Kinderarbeit) wurden von 110-135 der 193 Staaten der Erde ratifiziert.

Die Verbindung weltwirtschaftlicher Marktregulierung mit der Verantwortung für die Respektierung von Menschenrechten wird in der Praxis immer wieder in Frage gestellt, indem von dieser Verbindung a) Wettbewerbsnachteile befürchtet werden, b) indem verschiedene Menschenrechte miteinander in Konflikt stehen können (z.B. das Recht auf Entwicklung versus das Verbot missbräuchlicher Kinderarbeit) und c) indem Sozialklauseln nur ausgewählte Minimalstandards umfassen. Dem ist entgegenzuhalten, dass a) Menschenrechte unveräusserlich sind und ihre Respektierung ethisch gesehen nicht der ökonomischen oder politischen Opportunität unterworfen sein können, b) bei Konflikten zwischen Menschenrechten situationsethisch eine Güterabwägung vorzunehmen ist und c) die Menschenrechte zwar unteilbar sind, dies aber kein Grund ist, nicht mit spezifischen Massnahmen wie Sozialklauseln spezifische Menschenrechte zu schützen (vorausgesetzt, dass diese Massnahmen nicht verabsolutiert werden).

3. UNDP: Human Development Report, 1994, 90-226. Daly, H./ Cobb, J.B.: For the Common Good: Redirecting the Economy Toward Community, the Environment and a Sustainable Future, Boston 1989. 


\section{Kohärenz bedeutet Mitverantwortung für alle Lebensbereiche}

Menschliches Handeln kann nie widerspruchsfrei sein. Anzustreben ist aber, dass es widerspruchsärmer wird. Deshalb sind Menschenrechts- und Aussenwirtschaftspolitik, Aussenpolitik und Sozialpolitik, Umwelt- und Handelspolitik etc. im Dienste einer glaubwürdigen, kohärenten Politik miteinander in Verbindung zu setzen. Entsprechend muss die Geschäftspolitik einer internationalen Unternehmung in einem Entwicklungsland jener in einem Industrieland entsprechen. Die Respektierung sozialer Minimalstandards im nationalen wie internationalen wirtschaftlichen Handeln ist ein Ausdruck und eine Bedingung für kohärentes Handeln. Das Kohärenzanliegen ist national ${ }^{4}$ wie multilateral ${ }^{5}$ wie auch unternehmensethisch bedeutsam.

\section{Gerechtigkeit zielt auf die Verminderung des Wohlstandsgefälles}

Wirtschaftsethik misst wirtschaftliches Handeln selbstverständlich immer auch am Gerechtigkeitskriterium. Nur: was ist gerecht? Aus der sehr reichen theologischen und wirtschaftsethischen Debatte um das Gerechtigkeitsverständnis sei in der hier nötigen Kürze nur ein Aspekt hervorgehoben: John Rawls formulierte zwei berühmt gewordene und auch wirtschaftsethisch breit rezipierte Gerechtigkeitsgrundsätze. Der „Erste Grundsatz: Jedermann hat gleiches Recht auf das umfangreichste Gesamtsystem gleicher Grundfreiheiten, das für alle möglich ist" ${ }^{46}$, spiegelt sich in GATTNTO als einem weltumfassenden System gleicher Handelsfreiheiten ${ }^{7}$. Dieses Prinzip ist nun aber von Rawls zweitem Grundsatz nicht zu trennen: „Soziale und wirtschaftliche Ungleichheiten müssen folgendermassen beschaffen sein: a) sie müssen den am wenigsten Begünstigten den grösstmöglichen Vorteil bringen..." 8 . Vereinfacht gesagt: Gerecht ist, was die Schwächsten am meisten schützt und stärkt.

4. So will sich die offizielle schweizerische Aussenpolitik orientieren an der „Kohärenz ... zwischen Innen- und Aussenpolitik, ... zwischen verschiedenen Teilbereichen der Aussenpolitik, ... zwischen Zielen und Mitteln der Aussenpolitik..." (Bericht des Bundesrates über die Aussenpolitik der Schweiz in den 90er Jahren, vom 29. Nov. 1993, 45). Vorschläge zu einer kohärenten schweizerischen Entwicklungspolitik finden sich bei Gerster, R.: Nord-Süd-Politik: abschreiben oder investieren? Perspektiven der schweizerischen Entwicklungszusammenarbeit, Zürich 1995, 63-68.

5. Vgl. z.B. Gemeinsame Konferenz Kirche und Entwicklung GKKE/ European Ecumenical Organisation for Development EECOD: Towards Coherence in North/ South Policy: the Role of the European Union. Report from a Seminar in Brussels, 19-21 January 1994.

6. Rawls, J.: Eine Theorie der Gerechtigkeit, Frankfurt a.M. 1979, 336.

7. Rawls bezieht diesen Grundsatz primär auf die politischen Freiheiten in der Demokratie (ebd. 223-290), sekundär auch auf die politische Ökonomie (z.B. ebd., 306ff).

8. Ebd., 336. 
Der internationale Handel lebt nun aber ökonomisch gesehen vom Gefälle der Produktionskosten zwischen verschiedenen Standorten (Rohstoffe, Löhne, soziale Standards etc.). Er muss aber zugleich auf den Abbau dieser Gefälle hinarbeiten, wenn er die erwähnten Kriterien menschengerechter Entwicklung ernst nehmen will.

\section{Der Schutz der Schwachen darf nicht wegen Standortvorteilen missachtet werden}

Sozialklauseln werden von Regierungen des Südens mit dem Hinweis bekämpft, damit würden ihre Standortvorteile als Billiglohnländer im Welthandel geschmälert. Faktisch werden damit oft Eigeninteressen der Führungsschichten zulasten der Schwachen ihres Landes verteidigt. Umgekehrt werden Sozialklauseln von Regierungen und Unternehmungen des Nordens teilweise mit protektionistischen Absichten bejaht ${ }^{9}$ und damit Eigeninteressen der reichen Länder zulasten der wirtschaftlich schwächeren Länder verteidigt.

Die ethisch entscheidende Frage ist, wer von Standortvorteilen im Welthandel profitiert, einige Wenige oder die breite Bevölkerung. Standortvorteile dürfen nicht zulasten der Schwachen gehen. Andererseits bedeutet Schutz von Schwachen nicht Aufhebung des Wettbewerbs, der weniger effiziente und leistungsfähige Marktteilnehmer ausschaltet. Solidarität und Wettbewerb schliessen sich nicht aus, worauf der Ökonom Hermann Sautter kürzlich zu Recht hingewiesen hat ${ }^{10}$.

\section{Freiheit und Gerechtigkeit bedingen sich gegenseitig}

Die Dynamisierung der Weltwirtschaft wird durch die Liberalisierung des Welthandels angestrebt. Damit tritt der Wert der Freiheit erneut an erste Stelle der wirtschaftlichen Wertordnung. Freiheit ist ein wichtiger Grundwert. Jeder Wert wird aber pervertiert und zu einem Unwert, wenn er verabsolutiert wird. Verabsolutierte Freiheit führt zur Schrankenlosigkeit und zum Tod der Schwächeren, verabsolutierte Gerechtigkeit führt zur Gleichmacherei und zum totalitären Staat. Grundwerte bleiben nur so lange human, als sie in einem Gleichgewicht mit andern Grundwerten stehen. Freiheit ermöglicht menschengerechte Entwicklung, wenn sie mit Gerechtigkeit verbunden ist. Gerechtigkeit ermöglicht menschenwürdige Entwicklung, wenn sie Freiheit zulässt. Bisher hat die Liberalisierung des Welthandels die Ungleichheiten und Unge-

9. Ökonomische Untersuchungen weisen darauf hin, dass Arbeitslosigkeit im Norden weniger durch Arbeitsplatzverlagerung in den Süden als durch technologische Innovation verursacht ist. Allfällige Hoffnungen, Sozialklauseln hätten protektionistische Wirkungen und könnten damit Arbeitsplätze im Norden sichern, sind deshalb mit Vorsicht aufzunehmen.

10. Sautter, H.: Moralisches Urteil und ökonomisches Kalkül, Zeitschrift für Evangelische Ethik 39, 1995, 132-136. 
rechtigkeiten in der Weltwirtschaft nicht verringern können. Deshalb braucht der liberale Welthandel soziale Minimalstandards als Ergänzung.

\section{Soziale und wirtschaftliche Entwicklung bedingen sich gegenseitig}

Unter den gegenüber Sozialklauseln kritischen Positionen wird immer wieder das Argument erwähnt, „sozialer Fortschritt wird eher durch Handelserleichterungen als durch Handelsbeschränkungen gefördert"11, und der wirtschaftliche Aufschwung sei Voraussetzung für die soziale Entwicklung und die Finanzierung eines Sozialstaates. Welche sozialen Faktoren sind Voraussetzung der wirtschaftlichen Entwicklung, und welche sind eine Folge davon? Empirische volkswirtschaftliche Untersuchungen zu dieser Frage ${ }^{12}$ kommen zu verschiedenen Ergebnissen, doch scheint vieles darauf hinzudeuten, dass die soziale Entwicklung sowohl eine Voraussetzung für eine dauerhafte wirtschaftliche Entwicklung (im Süden wie im Norden) ist und zugleich deren Folge. Soziale Entwicklung erhöht Produktivität, Effizienz und Akzeptanz der Wirtschaft. Höhere Löhne und Heranbildung von Mittelschichten steigern die Kaufkraft, bessere Bildung stärkt die Wettbewerbsfähigkeit, Sozialpartnerschaft stärkt die soziale und politische Stabilität, die gerechte Verteilung des Bodens unter viele und das Recht von Frauen, Boden selbst besitzen zu können, erhöht die Produktivität. Es ist zu vermuten, dass Sozial- wie Ökoklauseln vermutlich die Wettbewerbsfähigkeit - zumindest mittelfristig - nicht vermindern, sondern fördern. Sie entsprechen damit nicht nur den Menschenrechten, sondern auch der ökonomischen Vernunft.

\section{Die Preise müssen die Wahrheit sagen - ökologisch und sozial}

„Die Preise müssen die Wahrheit sagen“ (E. U. von Weizsäcker). Diese Erkenntnis ist in der Umweltpolitik und -ethik nachgerade anerkannt, wenn auch noch lange nicht verwirklicht. Die ökologischen Kosten eines Produktes (von Ressourcennutzung, Abfällen, Schäden, bis zur Vorsorge für künftige Generationen), die bisher weitgehend als externe Kosten der Allgemeinheit aufgebürdet wurden, gilt es zu internalisieren. Die heutigen Preise geben erst etwa ein Fünftel oder ein Zehntel der ökologischen Wahrheit wieder. Ähnliches gilt für viele soziale Kosten besonders im Süden, aber auch teilweise im Norden. Die Preise müssen die Wahrheit über die sozialen Kosten sagen. Gesundheits-

11. Grossmann H./ Koopmann, G.: Sozialstandards im internationalen Handel? Wirtschaftsdienst Nov. 1994, 585-591 (591).

12. Empirische Ergebnisse sind von der Wirtschaftsethik zu berücksichtigen, was hier nur andeutungsweise geschehen kann: vgl. z.B. OECD: Trade and Labor Standards. A Review of the Issues, Paris, Mai 1995;. Wood, A.: North-South trade, employment and inequality. Changing fortunes in a skill-driven world, Oxford 1994; Charnovitz, St.: The Influence of International Labor Standards on the World Trading Regime: A Historical Overview, International Labor Review, Sept.-Oct. 1987. 
schädigungen durch den Arbeitsplatz fallen als Gesundheitskosten beim Staat an (sie entsprechen ökologisch der Übernutzung der Ressourcen, hier der human ressources), eine gute Ausbildung der Arbeitenden zu verhindern, heisst, den Graben zum Know how der Industrieländer zu vergrössern (dies entspricht ökologisch der mangelnden Vorsorge für künttige Generationen). $\mathrm{Zu}$ den sozialen Kosten gehören insbesondere auch die geschlechtsspezifischen. Frauen tragen die sozialen Kosten durch Doppelbelastung, tiefere Löhne, Verantwortung für die Gesundheit der Familie usw. oft stärker als Männer.

\section{Rechtlich verbindliche Instrumente zur Durchsetzung des Ethos sozialer Minimalstandards sind nötig}

Wie lassen sich wirtschaftsethische Normen wie soziale Minimalstandards verbindlich umsetzen? Eine breite Palette von Mitteln wird angewandt. Die ethische Erziehung und die Überzeugungsarbeit durch Gewissensbildung und vernünftige Argumentation im Dialog sind Wege, die auf Kooperation und Partizipation beruhen und dem christlichen Bild der liebenden, sich als gleichwertige Partner respektierenden Menschen entspricht. Solche partizipative Kooperation kann zu freiwilliger Einhaltung der Normen wie auch zu gemeinsam vereinbartem verbindlichem Recht führen. Das Ethos der Gerechtigkeit, des Schutzes der Schwachen usw. ist nicht nur, aber auch, mit Hilfe des Rechts zu verwirklichen.

Auf nationaler Ebene ist längst anerkannt, dass ethische Normen sozialverträglichen Wirtschaftens auch rechtlich verbindlich umgesetzt werden sollen. Im Welthandel mit seiner Entgrenzung nationaler Märkte bestehen diesbezüglich deutliche Defizite. Hauptinstrument z.B. innerhalb der IAO ist der moralische und mediale Druck, der zur Einhaltung freiwillig und kooperativ vereinbarter Konventionen führen soll. Wenn man bedenkt, mit welchem Nachdruck sich Regierungsvertreter bemühen, im Rahmen der IAO in den Länderberichten des Normenanwendungsausschusses Anmerkungen gegen das eigene Land zu vermeiden, dann wird die Bedeutung moralischen Drucks nicht zu unterschätzen sein.

Dennoch genügen mit der raschen Ausweitung internationaler Wirtschaftsaktivitäten Freiwilligkeit und moralischer Druck nicht mehr. Rechtsverbindliche Belohnungen oder Bestrafungen bei Einhaltung oder Verletzung fundamentaler Menschenrechte sind ethisch gesehen nicht nur national, sondern auch international zu unterstützen. Bestrafung geschieht z.B. durch Sanktionen. Es ist ethisch stossend und ökonomisch unbegründet, dass handelspolitische Sanktionen im Rahmen von GATT/WTO zulässig sind, wenn Exportländer einen Wettbewerbsvorteil durch künstliche Verbilligung ihrer Güter mittels Subventionen erreichen wollen, solche Sanktionen bis jetzt aber nicht erlaubt sind, wenn ein Wettbewerbsvorteil durch krasse Verstösse gegen grundlegende Arbeitnehmerrechte als Menschenrechte ergattert wird. Vom Kriterium der Kohärenz her sind Handelssanktionen gegen die Verlet- 
zung sozialer Minimalstandards deshalb nicht von vornherein abzulehnen ${ }^{13}$.

Im Sinne positiver Massnahmen stehen Belohnungen im Sinne positiver Massnahmen statt Sanktionen z.B. bei den Bemühungen der EU im Vordergrund, Zollpräferenzen für Exporte jenen Entwicklungsländern zu gewähren, die Sozialklauseln respektieren. Solche Konditionalität hat allerdings den Nachteil, dass sie nicht gemeinsam ausgehandelt, sondern einseitig bestimmt wird.

\section{Das Zusammenspiel verschiedener Handlungsebenen ist für die Durchsetzung sozialer Minimalstandards nötig}

So wie innerhalb der Ethik die Individual-, Personal- und Sozialethik und innerhalb der Wirtschaftsethik die ordnungspolitischen, unternehmensethischen und konsumentenethischen Aspekte zusammengehören, so braucht es auch für die Sozialverträglichkeit des Welthandels das Zusammenspiel verschiedener Handlungsebenen, die nicht gegeneinander ausgespielt werden dürfen: Soziale Schutzmechanismen werden vom Gleichbehandlungskriterium her primär multilateral durch WTO und IAO angestrebt, sind aber auch in bilateralen Handelsverträgen zu prüfen. In Ergänzung (nicht als Ersatz) sind zudem freiwillige Vereinbarungen zwischen Unternehmungen und Nichtregierungsorganisationen (wie Hilfswerken, FairTrade-Organisationen, Konsumentenorganisationen) bedeutsam, weil sie mit grösserer Wahrscheinlichkeit und rascher umgesetzt werden können als staatliche Vereinbarungen, und weil sie die konsumentennahe Bewusstseinsbildung fördern. Der rasche Aufschwung des Fairen Handels und die in der Schweiz initiierte Sozialklausel zwischen Migros und Del Monte Philippinen unter Beteiligung von Hilfswerken sind Beispiele dafür ${ }^{14}$. Dabei können produktbezogene, firmenbezogene, branchenbezogene und landesbezogene Sozialklauseln unterschieden werden. Häufig haben individual- oder unternehmensethische Ansätze Vorläuferfunktion, damit schliesslich ordnungspolitische Regelungen mehrheitsfähig werden.

13. So heisst es in den "Leitlinien für die internationale Geschäftstätigkeit“ von 1980 des schweizerischen „Gesprächskreises Kirche-Wirtschaft“ im Abschnitt über „Wirtschaftliche Beziehungen zu Ländern mit totalitären und rassistischen Systemen in Abschnitt 4.4: „Wirtschaftsbeziehungen sind zu überprüfen, wenn sie das Unternehmen zu einem Verhalten zwingen, das in offensichtlichem Widerspruch zu fundamentalen, menschlichen und gesellschaftlichen Werten steht."

14. Vgl. dazu: Lanz, Ch.: Testfall Ananas. Die Sozialklausel Migros/DelMonte, Bern 1987; Stückelberger, Ch.: Sozialklauseln für fairen Welthandel. Neue Schritte mit der Sozialklausel Migros/ Del Monte, Reformiertes Forum 35, Sept 1994, 3-4; Egger, M.: Clause sociale. Un nouveau départ, Terre Nouvelle (Lausanne), nov.-déc. 1994, $22 f$. Report of the Second Migros-Del Monte Social Clause Monitoring Panel, 60 p., nov1995, Manila/Bern. 